\title{
Anticipation of Age-of-Onset in Familial Amyloidotic Polyneuropathy and Its Pathogenesis
}

Recent advances in molecular biology have suggested that a genetic and clinical heterogeneity exists in transthyretin (TTR)associated amyloidosis. To date, amino acid substitutions at about 40 positions have been characterized both by protein structural data and DNA analysis. A variety of clinical syndromes have been associated with these several TTR mutations (1).

Methionine-30 TTR amyloidosis is the most common type of Familial Amyloidotic Polyneuropathy (FAP); it is termed FAP type I. While first described in families in northern Portugal by Andrade (2), it is also present in Japan, Sweden, Greece, Cypras, Majorca, Brazil, Turkey, and in the United States. The disease is a typical autosomal dominant disorder with prominent peripheral and autonomic nerve involvement.

A peripheral neuropathy starts in the lower extremities and progresses cephalad. The upper extremities are involved later in the disease and the cranial nerves may also be involved. The first symptoms of FAP type I may be bowel dysfunction and/or impotence.

The clinical heterogeneity extends to the onset of the disease. In Met 30 amyloidosis, the onset is very variable among patients, ranging from the 3 rd decade to the 7 th decade or even later.

The mean age of onset of the clinical syndrome in Portuguese patients is 32 for males, and 33 for females. Late onset may occur in some patients with symptoms not apparent until the 6 th or 7 th decade. In Japanese patients, the mean age of onset is 32 years, and in the Swedish kindreds, the mean age of onset is 58. Gene dosage does not influence the onset, as homozygous individuals can be asymptomatic late in life. In TTR amyloidosis, it is clear that amyloid formation precedes the onset of clinical symptoms, however it is difficult to assess the timing relationship between the two events. At this point, the intervening factors in amyloidogenesis are largely unknown, but it is well known that there is a multifactorial process where possible hypotheses are not conclusive.

\section{Age-of-onset and paternal transmission}

Despite its autosomal dominant mode of inheritance, women are known to be less often affected with FAP-I, and to have a later onset than men. Sequeiros et al (3) studied the sex ratio and age-of-onset distribution in 1,072 Portuguese patients. There were 625 men and 447 women affected with a sex ratio of 1.45:1. Among all asymptomatic carriers of Met-30TTR, 334 were women and 272 were men $(0.81: 1)$. The sex ratio for 1,678 (manifesting and non-manifesting) carriers was 1.15:1. Mean age-of-onset was 34.8 in women and 31.7 years in men. This reflects different age-of-onset distributions, with overall later onset and less affected women.

Sousa et al (4) further studied the consequences of age-ofonset of both sexes and that of the transmitting parent in Portuguese patients: 505 inherited the FAP gene from the mother, and 429 from the father. Men affected with FAP had, on average, an earlier onset than women. The mean age-ofonset was higher for children of transmitting fathers than of transmitting mothers, in any case, daughters have a higher mean age-of-onset. Thus, sons of affected mothers had the earliest onset (29.3), while daughters of affected fathers seemed to be the more "protected" (35.1 years) from the effects of the FAP gene. Both studies postulated a possible role of multifactorial inheritance of genetic modifiers, with a different threshold for each sex.

\section{Anticipation of age-of-onset in FAP-I}

Among Portuguese FAP patients, no late-onset child (onset at or after age 52 years) has ever been found to descend from a classic-onset parent (onset before age 40). Late-onset patients, however, often have classic-onset children. Sousa et al (4) looked at the transmission of age-of-onset (from affected parents to their offspring) through pedigree analysis. The offspring of late-onset patients usually have an earlier onset than their parents. Sons of affected mothers (who, on average, had FAP 10.9 years earlier than their mothers), and daughters of affected fathers (who had onset 1.3 years later than their fathers) showed the extreme means.

Drugge, et al (5) reported the results of a pedigree analysis on FAP-I in a Swedish family, using Swedish historical archives. The population studied included 239 patients: 109 patients were linked to five large pedigrees and 80 patients belonged to 30 smaller pedigrees or nuclear families. They found differences in mean ages of onset between the different pedigrees, although there was a considerable variation within the pedigrees. There was a tendency for later ages of onset among older generations than younger ones: descendants of affected mothers seemed to be more prone to an earlier age of onset than descendants of affected fathers. Furthermore, there seems to be a tendency for earlier ages of onset among patients with a carrier mother than a carrier father.

Tashima, et al (6) studied the age of onset in 33 alive FAP patients (1982-1994) confirmed by clinical and genetic inves- 
tigation including DNA method. They also investigated the clinical syndromes and the age of onset of 20 deceased FAP patients from 1920 to 1960 . They focused on the correlation between the age of onset and their birth year in calendar year. They found that the age of onset in Japanese FAP patients was younger year by year. This tendency was more apparent in female FAP patients than in male patients. A typical family showed the tendency of anticipation in 3 generations. In their study, the tested number of the FAP patients was small, and showed only one pedigree. The accuracy of the information on the initial symptoms in deceased FAP patients seemed to be insufficient and not conclusive. Further study is necessary to confirm their data in other pedigrees of Japanese FAP patients.

\section{Pathogenesis}

The pathogenesis of FAP is still unknown. The reason for the different age of onset remains unclear, but this fact suggests that the amyloid formation in FAP might not only be associated with the serum levels of variant TTR but also with other unknown factors. In 1995, the Welfare Ministry of Japan reported that the average life expectancy in Japanese is 76.57 years in males and 82.98 years in females. Comparison of these figures with those at the beginning of the 20th century (1901) shows an increase of 33.3 years and 38.7 years, respectively. The Japanese now have the longest life expectancy in the world. The major reason for this marked increase seems to be, in addition to advances in medicine, improvements in environment and nutrition resulting from economic growth in Japan.

In Japan, many FAP patients live in Kumamoto and Nagano prefectures; good food, comfortable life style, and good sani- tary conditions may have an affect on the onset of the disease. However, no crucial factor has as yet been found related to the pathogenesis of FAP. Anticipation of the age-of-onset in FAP awaits further study.

$$
\begin{array}{r}
\text { Shukuro ARAKI, MD } \\
\text { Professor Emeritus, } \\
\text { Kumamoto University. }
\end{array}
$$

\section{References}

1) Araki S, Ikegawa S. Transthyretin and Apo A-I amyloidosis. in: Amyloid and Amyloidosis 1993, Kisilevsky R, Benson MD, Frangione B, Gauldie J, Mucle TJ, Young ID, Eds. Parthenon Publishing, New York, 1994, pp. 422.

2) Andrade C. A peculiar form of peripheral neuropathy: Familial atypical generalized amyloidosis with special involvement of the peripheral nerves. Brain 75: 408, 1952.

3) Sequeiros J, Sousa A, Coelko I. Sex differences and age-dependent penetrance in FAP-Type I. in: Amyloid and Amyloidosis 1990, Natvig JB, Forre O, Husby G, Husebekk A, Skogen B, Sletten K, Westermark P, Eds. Kluwer Academic Publishers, Dordrecht, 1991, pp. 687.

4) Sousa A, Coelho T, Lobato L, Sequeiros J. Anticipation of age-of-onset in familial amyloidotic polyneuropathy (Portuguese Type). in: Amyloid and Amyloidosis 1990, Natvig JB, Forre O, Husby G, Husebekk A, Skogen B, Sletten K, Westermark P, Eds. Kluwer Academic Publishers, Dordrecht, 1991, pp. 694.

5) Drugge U, Anderson R, Chizari F, et al. Familial amyloidotic polyneuropathy in Sweden, a pedigree study. J Med Genet 30: 388, 1993.

6) Tashima K, Ando Y, Tanaka Y, Uchino M, Ando M. Change in the age of onset in patients with familial amyloidotic polyneuropathy type I. Intern Med 34: 748, 1995. 